\title{
ГРАНИЧНЫЕ ЗАДАЧИ ДЛЯ СЛАБО НАГРУЖЕННОГО ОПЕРАТОРА ГИПЕРБОЛИЧЕСКОГО УРАВНЕНИЯ ВТОРОГО ПОРЯДКА В ЦИЛИНДРИЧЕСКОЙ ОБЛАСТИ
}

\author{
Институт математики НАН Беларуси, Минск, Беларусь \\ korzyuk@bsu.by; Kozlovskaja@bsu.by; muvasharhan@gmail.com
}

\begin{abstract}
В сообщении рассмотрены три смешанные задачи в цилиндрической области для линейного гиперболического уравнения второго порядка, оператор которого слабо нагружен оператором такого же вида. Оператор, который нагружает исходный, имеет достаточно малые коэффициенты. Это требование выражается через оценки характеристического полинома. Изучение задач сводится к операторным уравнениям, которые рассмотрены в подходящих функциональных пространствах. Методами функционального анализа доказываются существование и единственность сильных решений.

Ключевые слова: дифференциальные уравнения, метод энергетических неравенств, операторы осреднения с переменным шагом, нагруженный оператор.
\end{abstract}

\section{I. KORZYUK, M. T. DZHENALIEV, I. S. KOZLOVSKAYA}

\section{BOUNDARY PROBLEMS FOR A WEAKLY LOADED OPERATOR OF THE SECOND-ORDER HYPERBOLIC EQUATION IN THE CYLINDRICAL AREA}

\author{
Institute of Mathematics of the National Academy of Sciences of Belarus, Minsk, Belarus \\ korzyuk@bsu.by; Kozlovskaja@bsu.by; muvasharhan@gmail.com
}

This article considers three mixed problems in the cylindrical area for the second-order linear hyperbolic equation with an operator which is weakly loaded with an operator of the same type. Coefficients of the loading operator are small enough. This demand is expressed through the estimates of the characteristic polynomial. The research of problems reduces to solving operator equations in suitable functional spaces. Existence and uniqueness of strong solutions were proved with the use of functional analysis methods.

Keywords: differential equations, energy inequality method, mollifiers with variable step, loaded operator.

Введение. Изучение граничных задач для нагруженных уравнений связано с многочисленными приложениями. Такого рода задачи являются объектом исследования во многих работах [1-9].

Следует отметить, что в упомянутых работах, в основном, рассмотрены граничные задачи для нагруженного уравнения теплопроводности. В данной работе предложена попытка сделать это для уравнения гиперболического типа с использованием метода энергетических неравенств и операторов осреднения с переменным шагом [10; 11]. В [10] под названием обобщенно-классического решения смешанных задач для гиперболических уравнений (параграф 3.6) рассмотрены граничные задачи в цилиндрической области с условиями Дирихле, Неймана (с производной по конормали) и условиями через производные по касательным направлениям. В данной работе изучаются эти задачи для аналогичного уравнения со слабо нагруженным оператором.

Под названием «слабо нагруженный оператор» имеется в виду оператор, коэффициенты которого достаточно малы по сравнению с коэффициентами основного оператора уравнения. Это будет выражаться в процессе вывода энергетического неравенства и доказательства существования решений рассматриваемых задач.

(С) Корзюк В. И., Дженалиев М. Т., Козловская И. С., 2015. 
1. Постановка задачи. Относительно функции $u: \mathbb{R}^{n+1} \supset Q$ э $\mathbf{x} \rightarrow u(\mathbf{x}) \in \mathbb{R}$ в цилиндрической области $Q=(0, T) \times \Omega$ независимых переменных $\mathbf{x}=\left(x_{0}, x_{1}, \ldots, x_{n}\right)$ рассмотрим нагруженное линейное дифференциальное уравнение второго порядка

$$
L u(\mathbf{x})=\frac{\partial^{2} u(\mathbf{x})}{\partial x_{0}^{2}}-A^{(0)} u(\mathbf{x})-B^{(0)} u\left(t, \mathbf{x}^{\prime}\right)+A^{(1)} u(\mathbf{x})+B^{(1)} u\left(t, \mathbf{x}^{\prime}\right)=f(\mathbf{x}) .
$$

Здесь $\quad \mathbb{R}^{n+1}-(n+1)$-мерное $\quad$ евклидово пространство, $\quad A^{(0)} u(\mathbf{x})=\sum_{i, j=1}^{n} \frac{\partial}{\partial x_{i}}\left(a^{(i j)}(\mathbf{x}) \frac{\partial u(\mathbf{x})}{\partial x_{j}}\right)$, $A^{(1)} u(\mathbf{x})=\sum_{i=0}^{n} a^{(i)}(\mathbf{x}) \frac{\partial u(\mathbf{x})}{\partial x_{i}}+\tilde{a}(\mathbf{x}) u(\mathbf{x}), B^{(0)} u=\sum_{i, j=1}^{n} b^{(i j)}\left(t, \mathbf{x}^{\prime}\right) \frac{\partial^{2} u\left(t, \mathbf{x}^{\prime}\right)}{\partial x_{i} \partial x_{j}}, B^{(1)} u\left(t, \mathbf{x}^{\prime}\right)=\sum_{i=0}^{n} b^{(i)}\left(t, \mathbf{x}^{\prime}\right) \frac{\partial u\left(t, \mathbf{x}^{\prime}\right)}{\partial x_{i}}+$ $b^{(0)}\left(t, \mathbf{x}^{\prime}\right) u\left(t, \mathbf{x}^{\prime}\right), \Omega$ - ограниченная область $\mathbb{R}^{n}$ переменных $\mathbf{x}^{\prime} \in \mathbb{R}^{n}$ с кусочно гладкой границей $\partial \Omega$.

Предположим, что (1) является гиперболическим относительно направления $\boldsymbol{\eta}=(1,0,0, \ldots, 0)$. Для этого потребуем выполнения условия положительности квадратичной формы, составленной из коэффициентов $a^{(i j)}$ оператора $A^{(0)}$, т. е.

$$
\sum_{i, j=1}^{n} a^{(i j)}(\mathbf{x}) \xi_{i} \xi_{j} \geq \alpha^{(0)} \sum_{i=1}^{n} \xi_{i}^{2}=\alpha^{(0)}|\xi|^{2}
$$

для любого вектора $\xi=\left(\xi_{1}, \ldots, \xi_{n}\right) \in \mathbb{R}^{n}$ и некоторого положительного числа $\alpha^{(0)}$ равномерно относительно $\mathbf{x} \in \bar{\Omega}, \bar{\Omega}$ - замыкание области $\Omega$.

Коэффициенты оператора $A^{(0)}$ удовлетворяют относительно индексов условию симметрии $a^{(i j)}=a^{(j i)}$ для любых $i, j=\overline{1, n}$. Кроме того, $a^{(i j)} \in C^{2}(\bar{Q})$, а $a^{(k)}, \tilde{a} \in C^{1}(\bar{Q}), k=\overline{0, n}$, где $C(\bar{Q})$ - множество непрерывных функций, а $C^{s}(\bar{Q})(s=1,2)$ - множество непрерывно дифференцируемых функций до порядка $s$ включительно, заданных на замыкании $\bar{Q}$ области $Q$.

Отсюда из условия $a^{(i j)} \in C(\bar{Q})$ следует неравенство

$$
\sum_{i, j=1}^{n}\left(a^{(i j)}(\mathbf{x})\right) \xi_{i} \xi_{j} \leq \alpha^{(1)} \mid \xi^{2}
$$

для любого $\xi=\left(\xi_{1}, \ldots, \xi_{n}\right) \in \mathbb{R}^{n}$ и любой точки $x \in \bar{Q}$, где $\alpha^{(1)}-$ некоторое положительное число. Очевидно, имеется соотношение $\alpha^{(0)} \leq \alpha^{(1)}$ между константами $\alpha^{(j)}, j=0,1$. Для коэффициентов оператора $A^{(1)}$ из того, что $a^{(k)}, \tilde{a} \in C^{1}(\bar{Q}), k=\overline{0, n}$, справедливы оценки

$$
\sum_{k=0}^{n} a^{(k)} \xi_{k} \leq \alpha^{(2)}|\xi|^{2},|\tilde{a}(\mathbf{x})| \leq \alpha^{(3)}
$$

для всех $x \in \bar{Q}$, где $\alpha^{(j)}, j=2,3,-$ некоторые положительные константы.

Аналогичные условия имеют место относительно коэффициентов операторов $B^{(0)}$ и $B^{(1)}$. Выполняются условия симметрии $b^{(i j)}=b^{(j i)},(i, j=\overline{1, n}), b^{(i j)} \in C^{2}(\bar{\Omega}), b^{(i)} \in C^{1}(\Omega),(i=\overline{0, n})$ и неравенство

$$
\sum_{i, j=1}^{n} b^{(i j)}\left(t, \mathbf{x}^{\prime}\right) \xi_{i} \xi_{j} \leq \beta^{(0)} \mid \xi^{2}=\beta^{(0)} \sum_{i, j=1}^{n} \xi_{i j}^{2}
$$

где $\xi=\left(\xi_{11}, \ldots, \xi_{i j}, \ldots, \xi_{n n}\right), \beta^{(0)}-$ положительная постоянная.

Справедлива аналогичная оценка и для оператора $B^{(1)}$, а именно:

$$
\left(B^{(1)} u\left(t, \mathbf{x}^{\prime}\right)\right)^{2} \leq \beta^{(1)}\left[\sum_{i=0}^{n}\left(\frac{\partial u}{\partial x_{i}}\right)^{2}\left(t, \mathbf{x}^{\prime}\right)+u\left(t, \mathbf{x}^{\prime}\right)\right]
$$

для некоторого положительного числа $\beta^{(1)}$ и любых функций $u \in C^{1}(\bar{Q})$.

К уравнению (1) присоединяются условия Коши

$$
l_{0} u=u\left(0, \mathbf{x}^{\prime}\right)=\varphi\left(\mathbf{x}^{\prime}\right), l_{1} u=\frac{\partial u\left(0, \mathbf{x}^{\prime}\right)}{\partial x_{0}}=\psi\left(\mathbf{x}^{\prime}\right)
$$


и одно из граничных условий

$$
\begin{gathered}
\left.u\right|_{\Gamma}=0, \\
\left.\frac{\partial u}{\partial \mathrm{N}}\right|_{\Gamma}=0, \\
\left.\frac{\partial u}{\partial \tau^{(1)}}\right|_{\Gamma}=\ldots=\left.\frac{\partial u}{\partial \tau^{(n)}}\right|_{\Gamma}=0, \int_{\partial \Omega} \sum_{i, j=1}^{n} a^{(i j)}(\mathbf{x}) v_{i} v_{j} \frac{\partial u}{\partial \mathbf{v}} u d s \leq 0,
\end{gathered}
$$

где $\Gamma=(0, T) \times \partial \Omega$,

$$
\left.\frac{\partial u}{\partial \mathbf{N}}\right|_{\Gamma}=\left.\sum_{i, j=1}^{n} a^{(i j)}(\mathbf{x}) \frac{\partial u}{\partial x_{j}} v_{i}\right|_{\Gamma},
$$

$\boldsymbol{v}=\left(v_{1}, \ldots, v_{n}\right)-$ единичный вектор внешней относительно $Q$ нормали, $\tau^{(1)}, \ldots, \tau^{(n)}-n$ линейно независимых касательных векторов, заданных почти всюду в точках гиперповерхности Г.

Таким образом, имеем следующие граничные (смешанные) задачи для нагруженного гиперболического уравнения (1):

первую смешанную задачу МР1 с граничными условиями Дирихле на боковой поверхности $\Gamma(1),(4),(5)$;

вторую смешанную задачу MP2 - (1), (4), (6);

третью смешанную задачу МP3 - (1), (4), (7).

Отметим, что могут рассматриваться смешанные задачи со смешанными на Г граничными условиями вида (5)-(7). В этом случае Г состоит из конечного числа частей и на каждой из этих частей задается какое-нибудь из условий (5)-(7).

2. Функциональные пространства и формулировка задач в операторном виде.

Задачи МP1-MP3 отличаются друг от друга граничными условиями (5)-(7). С учетом этих условий введем подмножества множества $C^{2}(\bar{Q})$.

Обозначим через $C^{2}(\bar{Q} ;(s)), s=5,6,7$, подмножество функций из $C^{2}(\bar{Q})$, которые удовлетворяют условиям $(s)$.

Для функций $w: \mathbb{R}^{n} \supset \Omega$ э $\mathbf{x}^{\prime} \rightarrow w\left(\mathbf{x}^{\prime}\right) \in \mathbb{R}$, заданных на $\Omega^{(0)}=\left\{\mathbf{x} \in \bar{Q} \mid x_{0}=0\right\}$, рассмотрим сужения условий (5)-(7), а именно

$$
\begin{gathered}
\left.\frac{\partial w}{\partial \mathbf{N}}\right|_{\partial \Omega}=0,\left.\frac{\partial w}{\partial \mathbf{N}}\right|_{\partial \Omega}=0, \\
\left.\frac{\partial u}{\partial \tilde{\tau}^{(1)}}\right|_{\partial \Omega} ^{n} \sum_{i, j=1} a^{(i j)}\left(0, \mathbf{x}^{\prime}\right) v_{i} \frac{\partial w}{\partial x_{j}}, \\
=\ldots=\left.\frac{\partial u}{\partial \tilde{\tau}^{(n-1)}}\right|_{\partial \Omega}=0, \int_{\partial \Omega} \sum_{i, j=1}^{n} a^{(i j)}\left(0, \mathbf{x}^{\prime}\right) v_{i} v_{j} \frac{\partial w}{\partial v} w d s \leq 0,
\end{gathered}
$$

где $\tilde{\tau}^{(1)}, \ldots, \tilde{\tau}^{(n-1)}$ - линейно независимые касательные к гиперповерхности $\partial \Omega$ единичные векторы; $\boldsymbol{v}=\left(v_{1}, \ldots, v_{n}\right)-$ единичный вектор внешней относительно области $\Omega$ нормали в точках $\mathbf{x}^{\prime}$ гиперповерхности $\partial \Omega$.

Обозначим через $C^{2}(\Omega ;(s)), s=8,9,10$, подмножество функций из множества $C^{2}(\bar{\Omega})$, которые удовлетворяют условиям $(s)$.

Обозначим через $H^{2}(\Omega)$ пространство Соболева квадратично суммируемых по Лебегу вместе с квадратично суммируемыми обобщенными производными до второго порядка включительно функций, заданных в области $\Omega$. Пусть $H^{2}(\Omega,(s))(s=8,9,10)$ подпространства пространства $H^{2}(\Omega)$, элементы которого удовлетворяют граничным условиям $(s), s \in\{8,9,10\}$.

У с л о в и е. Граница $\partial \Omega$ является кусочно-гладкой и такой, что пространства $H^{2}(\Omega,(s))$ $(s=8,9,10)$ можно получить путем замыкания соответствуюших множеств $C^{2}(\bar{\Omega} ;(3, s))$ по норме пространства $H^{2}(\Omega)$.

Пусть $H^{1}(\Omega,(s)) \quad(s=8,9,10)$ замыкания соответствующих множеств $C^{2}(\bar{\Omega} ;(s))$ по норме пространства $H^{1}(\Omega)$. 
Для функций $u \in C^{2}(\bar{Q})$ рассмотрим норму, которая задается выражением

$$
\|u\|_{B}=\left\|\frac{\partial^{2} u}{\partial x_{0}^{2}}\right\|_{L_{2}(Q)}+\sup _{0 \leq x_{0} \leq T}\left(\|u\|_{H^{2}(\Omega)}+\sum_{i=1}^{n}\left\|\frac{\partial^{2} u}{\partial x_{0} \partial x_{i}}\right\|_{L_{2}(\Omega)}+\left\|\frac{\partial u}{\partial x_{0}}\right\|_{L_{2}(\Omega)}\right)\left(x_{0}\right) .
$$

Введем банаховы пространства $B^{(s)}(Q), s=\overline{1,3}$, путем замыкания множеств $C^{2}(\bar{Q} ;(s+4))$ по норме, определяемой выражением (11).

Обозначим через $\mathfrak{A}^{1}(Q)$ гильбертово пространство квадратично суммируемых по Лебегу функций $u$, для которых существуют обобщенные производные $\frac{\partial u}{\partial x_{i}} \in L_{2}(Q), i=\overline{1, n}$. Скалярное произведение и норма определяются выражениями

$$
(u, v)_{\mathfrak{A}^{1}(Q)}=(u, v)_{L_{2}(Q)}+\sum_{i=1}^{n}\left(\frac{\partial u}{\partial x_{i}}, \frac{\partial u}{\partial x_{i}}\right)_{L_{2}(Q)},\|u\|_{\mathfrak{A}^{1}(Q)}=(u, u)_{\mathfrak{A}^{1}(Q)}^{1 / 2}, u, v \in \mathfrak{A}^{1}(Q) .
$$

Пусть $\mathfrak{A}^{1}(Q)$ - подпространство пространства $\mathfrak{A}^{1}(Q)$, полученное замыканием множества $C^{2}(\bar{Q} ;(5))$ по норме (12).

Введем операторы $\mathbf{L}^{s}, s=\overline{1,3}$, которые определяются следующим образом:

$$
\mathbf{L}^{s}: B^{(s)}(Q) \ni u \rightarrow \mathbf{L}^{(s)} u=\left\{\mathcal{L} u, l_{0} u, l_{1} u\right\} \in \mathbf{H}^{s}=\stackrel{\mathfrak{A}}{1}^{1}(Q) \times H^{2}(\Omega ;(k)) \times H^{1}(\Omega ;(k)), k=8,9,10 .
$$

Теперь каждую задачу MPs, $s=\overline{1,3}$, можно рассматривать как операторное уравнение

$$
\mathbf{L}^{(s)} u=\mathbf{F}^{(s)}, s=\overline{1,3},
$$

с областью определения $\mathcal{D}\left(\mathbf{L}^{(s)}\right)=\left\{C^{2}(\bar{Q} ;(s+4))|\mathcal{L} u|_{\Gamma}=0\right\}$, где $\mathbf{F}^{(s)}=\left\{f(x), \varphi\left(\mathbf{x}^{\prime}\right), \psi\left(\mathbf{x}^{\prime}\right)\right\} \in \mathbf{H}^{(s)}$.

3. Энергетические неравенства. Докажем энергетические неравенства для операторов $\mathbf{L}^{s}$, $s=1,3$, уравнений (13) для функций из $\mathcal{D}\left(\mathbf{L}^{(s)}\right)$. На основании этих неравенств вводится расширение операторов $\mathbf{L}^{s}$, до замкнутых $\overline{\mathbf{L}^{(s)}}$, путем замыкания, а затем доказываются теоремы существования и единственности сильных решений.

Т е о р е м а 1. Пусть выполняется условие и ограничения гладкости в $n$. 1 на коэффиииенты уравнения (1). Тогда при достаточно малых $\beta^{(0)}$ и $\beta^{(1)}$ справедливы энергетические неравенства

$$
\|u\|_{B} \leq c\left\|\mathbf{L}^{(s)} u\right\|_{\mathbf{H}^{(s)}}
$$

для любой функичи и из соответствующей области $\mathscr{D}\left(\mathbf{L}^{(s)}\right)$ и каждого оператора $\mathbf{L}^{(1)}, \mathbf{L}^{(2)} u \mathbf{L}^{(3)}$, где положительная константа с не зависит от и. Для доказательства теоремы 1 рассматривается выражение $\mathcal{L} u \frac{\partial}{\partial x_{0}} A^{(0)}(u)$, которое предста-
вим в виде

$$
\begin{gathered}
2\left(L u \frac{\partial}{\partial x_{0}} A^{(0)}(u)\right)(\mathbf{x})=2 \sum_{i, j=1}^{n} \frac{\partial}{\partial x_{i}}\left(a^{(i j)} \frac{\partial^{2} u}{\partial x_{0}^{2}} \frac{\partial^{2} u}{\partial x_{0} \partial x_{j}}\right)(\mathbf{x})-\frac{\partial}{\partial x_{0}}\left(\mathfrak{A}^{(0)}(u, u)(\mathbf{x})\right)+ \\
\mathfrak{A}^{(1)}(u, u)(\mathbf{x})+2 \frac{\partial}{\partial x_{0}}\left(A^{(0)} u A^{(1)} u\right)(\mathbf{x})-\mathcal{B}^{(0)}(u, u)(t, \mathbf{x})+\mathcal{B}^{(1)}(u, u)(t, \mathbf{x}),
\end{gathered}
$$

где

$$
\begin{gathered}
\mathfrak{A}^{(0)}(u, u)(\mathbf{x})=\sum_{i, j=1}^{n} \frac{\partial}{\partial x_{i}}\left(a^{(i j)} \frac{\partial^{2} u}{\partial x_{0} \partial x_{i}} \frac{\partial^{2} u}{\partial x_{0} \partial x_{j}}\right)(\mathbf{x})+\left(A^{(0)} u\right)^{2}(\mathbf{x}) \\
\mathfrak{A}^{(1)}(u, u)(\mathbf{x})=\sum_{i, j=1}^{n}\left[\frac{\partial a^{(i j)}}{\partial x_{0}} \frac{\partial^{2} u}{\partial x_{0} \partial x_{i}} \frac{\partial^{2} u}{\partial x_{0} \partial x_{j}}+2 \frac{\partial^{2} u}{\partial x_{0}^{2}} \frac{\partial}{\partial x_{i}}\left(\frac{\partial a^{(i j)}}{\partial x_{0}} \frac{\partial u}{\partial x_{j}}\right)\right](\mathbf{x})-2\left(A^{(0)} u \frac{\partial}{\partial x_{0}} A^{(1)} u\right)(\mathbf{x}) \\
\mathcal{B}^{(0)}(u, u)=2 B^{(0)} u\left(t, \mathbf{x}^{\prime}\right) A^{(0)} u\left(\mathbf{x}^{\prime}\right) \\
\mathcal{B}^{(1)}(u, u)=2 B^{(1)} u\left(t, \mathbf{x}^{\prime}\right) A^{(0)} u\left(\mathbf{x}^{\prime}\right) .
\end{gathered}
$$


С другой стороны,

$$
\left(\mathcal{L} u \frac{\partial}{\partial x_{0}} A^{(0)} u\right)(\mathbf{x})=\sum_{i, j=1}^{n} \frac{\partial}{\partial x_{i}}\left(\mathcal{L} u \frac{\partial}{\partial x_{0}}\left(a^{(i j)} \frac{\partial u}{\partial x_{j}}\right)\right)(x)-\sum_{i, j=1}^{n} \frac{\partial \mathcal{L} u}{\partial x_{i}} \frac{\partial}{\partial x_{0}}\left(a^{(i j)} \frac{\partial u}{\partial x_{j}}\right)(\mathbf{x}) .
$$

Обозначим через $Q(\tau)$ подобласть области $Q$ высотой $\tau$, т. е. $Q^{(\tau)}=(0, \tau) \times \Omega, 0<\tau<T$. Равенство (15) интегрируем по подобласти $Q^{(\tau)}$ области $Q$, используя при этом граничное условие $(s+4)$ в зависимости от оператора $\mathbf{L}^{s}, s=\overline{1,3}$.

Затем в левых и правых частях полученных равенств делаем соответствующие оценки. В результате получим доказываемые неравенства (14).

4. Существование и единственность сильных решений смешанных задач MPs, $s=\overline{1,3}$. На основании критерия замыкаемости оператора непосредственной проверкой доказывается, что все операторы $\mathbf{L}^{(s)}: B^{(s)}(Q) \rightarrow \mathbf{H}^{(s)}$ допускают замыкания $\mathbf{L}^{(s)}$. В дальнейшем будем рассматривать операторы $\mathbf{L}^{(s)}[10]$.

О п р е д е л е н и е. Решения операторных уравнений

$$
\overline{\mathbf{L}^{(s)}} u=\mathbf{F}^{(s)}, \mathbf{F}^{(s)} \in \mathbf{H}^{(s)}, s=\overline{1,3},
$$

будем называть сильными решениями или в нашем случае пространств $B^{(s)}(Q)$ - обобщенноклассическими решениями смешанных задач MPs, $s=\overline{1,3}$.

Путем предельного перехода для операторов $\overline{\mathbf{L}^{(s)}}, s=\overline{1,3}$, из энергетического неравенства (14) получаем энергетическое неравенство

$$
\|u\|_{B} \leq c\left\|\overline{\mathbf{L}^{(s)}} u\right\|_{\mathbf{H}^{(s)}}
$$

для любых $u \in D\left(\overline{\mathbf{L}^{(s)}}\right)$, где положительная постоянная $c$ та же, что и в неравенстве (14).

Т е о р е м а 2. Пусть выполняется условие и ограничения гладкости в п. 2 на коэффициенты уравнения (1). Тогда для любых функиий $f \in \mathfrak{A}^{1}(Q), \varphi \in H^{2}(\Omega,(3, s)) u \psi \in H^{1}(\Omega,(3, s)), s=\overline{1,3}$, существует и единственно обобщенно-классическое решение $u \in B^{(s)}(Q)$ соответствующей смешанной задачи MPs $(s=\overline{1,3})$ и справедлива оценка

$$
\|u\|_{B} \leq c\left(\|f\|_{\mathfrak{A}^{1}(Q)}+\|\varphi\|_{H^{2}(\Omega)}+\|\psi\|_{H^{1}(\Omega)}\right) .
$$

Д о к а з а т е л ь с т в о. Единственность и оценка (17) для обобщенно-классического решения каждой задачи MPs, если оно существует, следуют из энергетического неравенства (16).

Как известно, [10, т. 3.2.2], доказательство существования сильного решения, согласно определению, сводится к доказательству равенства $\mathfrak{R}\left(\overline{\mathbf{L}^{(s)}}\right)=\overline{\mathfrak{R}\left(\mathbf{L}^{(s)}\right)}$, т. е. к доказательству плотности множества значений $\mathfrak{R}\left(\mathbf{L}^{(s)}\right)$ оператора $\mathbf{L}^{(s)}$ в пространстве $\mathbf{H}^{(s)}$. Доказательство данного утверждения сводится к следующему: равенство

$$
\left(\mathbf{L}^{(s)} u, \mathbf{v}\right)_{\mathbf{H}^{(s)}}=(\mathcal{L} u, v)_{\mathfrak{A}^{1}(Q)}+\left(l_{0} u, v^{(0)}\right)_{H^{2}(\Omega)}+\left(l_{1} u, v^{(1)}\right)_{H^{1}(\Omega)}=0
$$

для любой функции $u \in \mathscr{D}\left(\mathbf{L}^{(s)}\right)$ выполняется тогда и только тогда, если $v=0$ в $\mathfrak{A}^{1}(Q), v^{(0)}=0$ в $H^{2}(\Omega,(3, s))$ и $v^{(1)}=0$ в $H^{1}(\Omega,(3, s))$ для $s=\overline{1,3}$ в отдельности.

Обозначим через $\mathcal{L}_{0}$ главную часть оператора $\mathcal{L}$. Кроме того, через $\mathscr{D}\left(\mathbf{L}^{(s)}\right)$ обозначим подмножество множества $\mathcal{D}\left(\mathbf{L}^{(s)}\right)$, для элементов $u$ которого $l_{0} u=l_{1} u=0$. В наших обозначениях пусть $\stackrel{\circ}{\mathbf{L}_{0}^{(s)}}=\left\{\stackrel{\circ}{\mathcal{L}_{0}}, l_{0}, l_{1}\right\}$.

Теперь в частном случае уравнение (18) можно представить в виде 


$$
\left(\mathcal{L}_{0} u, v\right)_{\mathfrak{A}^{1}(Q)}=\left(\mathcal{L}_{0} u, v\right)_{L_{2}(Q)}+\sum_{j=1}^{n}\left(\frac{\partial}{\partial x_{j}} \mathcal{L}_{0} u, \frac{\partial}{\partial x_{j}} v\right)_{L_{2}(Q)}=0
$$

где $u$ - произвольная функция из $\stackrel{\circ}{\mathscr{D}}\left(\begin{array}{c}\stackrel{(s)}{(s)} \\ 0\end{array}\right)$. В равенстве (19) значения $\mathcal{L}_{0} u$ и $\partial / \partial x_{j} \mathcal{L}_{0} u$ линейно независимы. Поэтому, если множества $\left\{\mathcal{L}_{0} u \mid u \in \stackrel{\circ}{\mathscr{D}}\left(\begin{array}{c}\circ \\ \mathbf{L}\end{array}\right)\right\}$ и $\left\{\partial / \partial x_{j} \mathcal{L}_{0} u \mid u \in \stackrel{\circ}{\mathscr{D}}\left(\begin{array}{c}\circ \\ \mathbf{L}\end{array}\right)\right\}, j=\overline{1, n}$, являются плотными в $L_{2}(Q)$, то из равенства (19) следуют условия ортогональности для каждого слагаемого, т. е. равенства

$$
\left(\mathcal{L}_{0} u, v\right)_{L_{2}(Q)}=0,\left(\frac{\partial}{\partial x_{j}} \mathcal{L}_{0} u, \frac{\partial}{\partial x_{j}} v\right)_{L_{2}(Q)}=0, j=\overline{1, n} .
$$

Согласно утверждению 3.4 .5 [10], вместо равенств (20) можно рассматривать в эквивалентном виде равенства

$$
\left(\mathcal{J}_{(k)} \mathcal{L}_{0} u, v\right)_{L_{2}(Q)}=0,\left(J_{(k)} \frac{\partial}{\partial x_{j}} \mathcal{L}_{0} u, \frac{\partial}{\partial x_{j}} w^{(j)}\right)_{L_{2}(Q)}=0, j=\overline{1, n}
$$

которые выполняются для любой функции $u \in \stackrel{\circ}{\mathscr{D}}\left(\mathbf{L}_{0}^{(s)}\right)$ и некоторых элементов $v, w^{(j)}$ из пространства $L_{2}(Q)$. В (21) $J_{(k)}$ - соответствующие операторы осреднения с переменным шагом $[12-14 ; 10]$, учитывающие граничные условия на границе $\partial Q$ области $Q$ рассматриваемых задач MPs, $s=1,3$.

Таким образом, далее доказательство теоремы 2 базируется на доказательстве плотности множеств $\left\{J_{(k)} \mathcal{L}_{0} u \mid u \in \stackrel{\circ}{\mathscr{D}}\left(\mathbf{L}_{0}^{(s)}\right)\right\}$ и $\left\{J_{(k)} \frac{\partial}{\partial x_{j}} \mathcal{L}_{0} u \mid u \in \stackrel{\circ}{D}\left(\mathbf{L}_{0}^{(s)}\right)\right\}, j=\overline{1, n}$, в пространстве $L_{2}(Q)$. Сформулируем данный желаемый результат в виде леммы.

Л е м м а. Пусть выполняются условия теоремы 2. Тогда равенства (21) для элементов v, $w^{(j)}, j=\overline{1, n}$, из пространства $L_{2}(Q)$ и любых функиий и $\in \stackrel{\circ}{D}\left(\mathbf{L}_{0}^{(s)}\right)$ имеют место тогда и только тогда, когда $v=w^{(j)}=0$ в $L_{2}(Q)$.

Таким образом, из утверждения леммы, согласно утверждению 3.4 .5 из [10], множества $\left\{\mathcal{L}_{0} u \mid u \in \stackrel{\circ}{\mathcal{D}}\left(\mathbf{L}_{0}^{(s)}\right)\right\},\left\{\partial / \partial x_{j} \mathcal{L}_{0} u \mid u \in \stackrel{\circ}{\mathscr{D}}\left(\mathbf{L}_{0}^{(s)}\right)\right\}$ являются плотными множествами в $L_{2}(Q)$. Следовательно, как было сказано ранее, из равенства (19) следуют соотношения (20). А из леммы и (20) имеем $v=0, \partial / \partial x_{j} v=0$, т. е. $v=0$ в $\mathfrak{A}^{1}(Q)$.

Так как $v=0$ в $\mathfrak{A}^{1}(Q)$, то имеет место (19) и равенство (18) уже имеет вид

$$
\left(l_{0} u, v^{(0)}\right)_{H^{2}(\Omega)}+\left(l_{1} u, v^{(1)}\right)_{H^{1}(\Omega)}=0
$$

для любой функции $u \in \mathcal{D}\left(\mathbf{L}_{0}^{(s)}\right)$. Поскольку операторы $l_{0}$ и $l_{1}, \partial / \partial x_{j} l_{k}$ и $\partial^{2} / \partial x_{j} \partial x_{k} l_{0}, j, k=\overline{1, n}$, линейно независимы и множества их значений плотны в $L_{2}(\Omega)$ для $\mathscr{D}\left(l_{i}\right)=\mathscr{D}\left(\mathbf{L}^{(3)}\right)$, то $v^{(0)}=0$ в $H^{2}(\Omega,(3, s))$, а $v^{(1)}=0$ в $H^{1}(\Omega,(3, s))$ для $s=\overline{1,3}$.

В общем случае оператора $\mathbf{L}^{(s)}$ равенство $\widehat{R\left(\mathbf{L}^{(s)}\right)}=H$ можно доказать методом вывода энергетического неравенства для формально сопряженной задачи с помощью операторов осреднения с переменным шагом или методом продолжения по параметру [10, т. 3.2.3] или [11].

\section{Список использованной литературы}

1. Нахушев, А. М. Нагруженные уравнения и их приложения / А. М. Нахушев // Дифференц. уравнения. - 1983. T. 19, № 1. - C. 86-94. 
2. Нахушев, А. М. Уравнения математической биологии / А. М. Нахушев. - М., 1995.

3. Дженалиев, М. Т. К теории линейных краевых задач для нагруженных дифференциальных уравнений / М. Т. Дженалиев. - Алматы, 1995.

4. Дженалиев, М. T. О нагруженных уравнениях с периодическими граничными условиями / М. Т. Дженалиев // Дифференц. уравнения. - 2001. - Т. 37, № 1. - С. 48-54.

5. Кожанов, А. И. Об одном нелинейном нагруженном параболическом уравнении и о связанной с ним обратной задаче / А. И. Кожанов // Мат. заметки. - 2004. - Т. 76, вып. 6. - С. 84-91.

6. Дженалиев, М. Т. Граничные задачи для спектрально-нагруженных параболических операторов / М. Т. Дженалиев, М. И. Рамазанов // Неклассические уравнения математической физики. - Новосибирск, 2007. - С. $114-127$.

7. Дженалиев, M. T. Об одной граничной задаче для спектрально-нагруженного оператора теплопроводности. I/ М. Т. Дженалиев, М. И. Рамазанов // Дифференц. уравнения. - 2007. - Т. 43, № 4. - С. 498-508.

8. Дженалиев, М. Т. Об одной граничной задаче для спектрально-нагруженного оператора теплопроводности. II / М. Т. Дженалиев, М. И. Рамазанов // Дифференц. уравнения. - 2007. - Т. 43, № 6. - С. 788-794.

9. Дженалиев, М. Т. О граничной задаче для спектрально-нагруженного оператора теплопроводности / М. Т. Дженалиев, М. И. Рамазанов // Сиб. мат. журн. - 2006. - Т. 47, № 3. - С. 527-547.

10. Корзюк, В. И. Метод энергетических неравенств и операторов осреднения. Граничные задачи для дифференциальных уравнений с частными производными / В. И. Корзюк. - Минск: Изд. центр БГУ, 2013. - 460 с.

11. Корзюк, В. И. Уравнения математической физики / В. И. Корзюк. - Минск: Изд. центр БГУ, 2011. - 460 с.

12. Deny, J. Les espaces du type de Beppo Levi / J. Deny, J. L. Lions // Ann. Inst. Fourier. - 1953-1954. - Vol. 5. P. $305-370$

13. Burenkov, V. I. Sobolev Spaces on Domains / V. I. Burenkov. - Stuttgard, 1998. - 312 p.

14. Буренков, В. И. Приближение бесконечно дифференцируемыми функциями с сохранением граничных значений / В. И. Буренков // Тр. МИ АН СССР. - 1987. - Т. 180. - С. 68-70. 